\title{
Optical and Mechanical Excitation Thermography for Impact Response in Basalt-Carbon Hybrid Fiber-Reinforced Composite Laminates
}

\author{
Hai Zhang, Member, IEEE, Stefano Sfarra, Fabrizio Sarasini, Clemente Ibarra-Castanedo, Stefano Perilli, Henrique \\ Fernandes, Yuxia Duan, Jeroen Peeters, Nicolas P. Avdelidis and Xavier Maldague, Senior Member, IEEE,
}

\begin{abstract}
In this paper, optical and mechanical excitation thermography were used to investigate basalt fiber reinforced polymer (BFRP), carbon fiber reinforced polymer (CFRP) and basalt-carbon fiber hybrid specimens subjected to impact loading. Interestingly, two different hybrid structures including sandwichlike and intercalated stacking sequence were used. Pulsed phase thermography (PPT), principal component thermography (PCT) and partial least squares thermography (PLST) were used to process the thermographic data. X-ray computed tomography (CT) was used for validation. In addition, signal-to-noise ratio (SNR) analysis was used as a means of quantitatively comparing the thermographic results. Of particular interest, the depth information linked to Loadings in PLST was estimated for the first time. Finally, a reference was provided for taking advantage of different hybrids in view of special industrial applications.
\end{abstract}

Index Terms-Infrared thermography, Nondestructive testing, Hybrid composite, SNR analysis.

\section{INTRODUCTION}

$\mathbf{Q}$ UALITY control (QC) is playing an increasingly important role for modern industrial production. This enhances the need of advanced image inspection techniques [1]-[4]. Image inspection includes a wide group of analytical techniques used in science and industry to evaluate the properties of materials, components, or systems preventing potential damages after manufacturing or in service [5], [6]. During the manufacturing and applications of composite materials, besides employing advanced manufacturing techniques to raise

Manuscript received April 19, 2017; revised August 16, 2017; accepted August 21, 2017. This research is supported by the Canada Research Chair in Multipolar Infrared Vision (MiViM) and the Natural Sciences and Engineering Research Council (NSERC) Canada.

H. Zhang, C. Ibarra-Castanedo and X. Maldague are with the Dept. of Electrical and Computer Engineering, Computer Vision and Systems Laboratory, Laval University, Quebec, QC, G1V 0A6, Canada. E-mail: hai.zhang.1@ulaval.ca (H. Zhang)

S. Sfarra and S. Perilli are with the Dept. of Industrial and Information Engineering and Economics, Las.E.R. Laboratory, University of L'Aquila, Roio Poggio, AQ 67100, Italy; S. Sfarra is also with Tomsk Polytechnic University, 634028, Tomsk, Savinykh St., 7, Russia.

F. Sarasini is with the Dept. of Chemical Engineering Materials Environment \& UDR INSTM, Sapienza University of Rome, Via Eudossiana 18, Rome, I-00184, Italy.

H. Fernandes is with the Dept. of Computer Science, Federal University of Uberlandia, 2121 Avenida Joao Naves de Avila, Uberlandia, 38400-902, Brazil.

Y. Duan is with the School of Physics and Electronics, Central South University, 932 Lushan South Road, Changsha, 410083, China.

J. Peeters is with the Dept. of Electromechanical Engineering, University of Antwerp, Groenenborgerlaan 171, B-2020 Antwerp, Belgium.

N.P. Avdelidis is with the Dept. of Materials Science \& Engineering, National Technical University of Athens, 15780, Athens, Greece. the production rate, the utilization of reliable and cost-effective condition monitoring, fault diagnosis, nondestructive testing (NDT), and structural health monitoring is very important [7]. In this direction, the inspection of post-impact damages of industrial aerospace composite materials via image inspection techniques is becoming more and more common [8]-[10].

The infrared thermography (IRT) technique is based on the recording of images after or while thermally stimulating the inspected component and is gaining increasing attention in the recent years due to its fast inspection rate, contactless nature, spatial resolution and acquisition rate improvements of infrared cameras. In addition, the development of advanced image processing techniques plays an important role in its exponential increment [11]-[13]. IRT can be used to assess and predict the structural integrity beneath the surface by measuring the distribution of infrared radiation and converting the measurements into a temperature scale [14]. Among the whole set of experimental set-ups, optical excitation thermography has been applied due to its ability to retrieve quantitative information concerning the defects. In addition, mechanical excitation thermography is also attracting increasing attention due to the powerful excitation approach [15].

Given the high specific strength and stiffness, carbon fiber reinforced polymer composites (CFRP) are often used in the aerospace industry. However, their toughness is considerably low, therefore the impact damage resistance is not very high. Impact damage is one of the most important threats for the aerospace industry. Impact loading may occur during the process of manufacturing, assembly, maintenance, and operation. Impact damage can greatly affect the residual mechanical properties of CFRP even if the damage is barely visible at the surface [16]. One solution is represented by fiber hybridization (usually with high strain to failure fibers). In this regard, glass fiber is the best option mainly because it is inexpensive. Basalt fibers can be considered as an interesting alternative to glass fibers taking into account the mechanical properties and the environmental benefits [17]. This type of fiber, obtained from basalt rocks, has been demonstrated to be effective for the reinforcement of polymeric matrices [18].

An in-depth study of impact damage in hybrid composites composed of basalt and carbon fibers has not been well documented yet in the open literature centred on thermographic inspection. In this paper, optical and mechanical excitation thermography were used to inspect basalt fiber reinforced polymer laminate (BFRP), CFRP and basalt-carbon fiber 
hybrid specimens. Interestingly, two different hybrid structures including sandwich-like and intercalated (i.e., alternating sequence of basalt and carbon fabrics) were used. Pulsed phase thermography (PPT), partial least squares thermography (PLST) and principal component thermography (PCT) techniques were also used to process the raw thermographic data. $\mathrm{X}$-ray computed tomography (CT) was used for validation. In addition, signal-to-noise ratio (SNR) analysis was used as a means of quantitatively comparing the thermographic results. Of particular interest, the depth information linked to Loadings in PLST was estimated for the first time. Finally, a reference was provided for taking advantage of different hybrids in view of special industrial applications.

\section{MATERIALS}

Concerning the materials used in this work, the plain weave basalt (BAS 220.1270.P) and carbon (CC160) fabrics have a fiber areal weight of $220 \mathrm{~g} / \mathrm{m}^{2}$ and $160 \mathrm{~g} / \mathrm{m}^{2}$, respectively. Bi-component epoxy resin (EC157+W152 MR) was selected as the polymeric matrix. The specimens were manufactured by a Resin Transfer Moulding (RTM) system and were cured for $12 \mathrm{~h}$ at room temperature and $4 \mathrm{~h}$ at $70{ }^{\circ} \mathrm{C}$. All of the specimens have thirteen layers with similar overall fiber volume fraction of 0.32 , the thickness of $3.5 \mathrm{~mm}$ and the dimension of $180 \mathrm{~mm} \times 60 \mathrm{~mm}$.

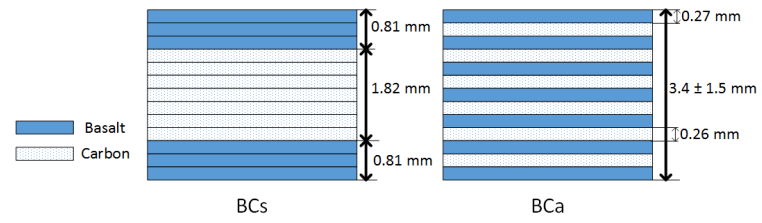

Fig. 1. Schematic diagram of basalt-carbon fiber hybrid structures.

The hybrid specimen (BCs) was stacked as a sandwich structure with seven carbon fiber layers (core) and three basalt fiber layers (skins) for each side. The hybrid specimen (BCa) has seven basalt fiber layers and six carbon fiber layers alternatively stacked, keeping basalt plies as outer layers on both sides. Fig. 1 shows the schematic diagram of the hybrid structures. Non-hybrid BFRP (B) and CFRP (C) specimens were also manufactured for comparative purposes. A falling dart impact machine (CEAST 9350) was used for low-velocity impact testing. All of the specimens were tested at 12.5 $\mathrm{J}$ by keeping constant the indenter mass $(6.929 \mathrm{~kg})$ with a hemispherical impact head (12.7 $\mathrm{mm}$ of diameter). The circular specimen holder has an external diameter of $60 \mathrm{~mm}$ and inner diameter of $40 \mathrm{~mm}$ [19].

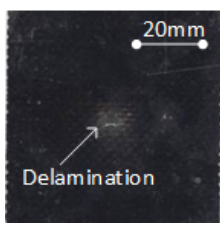

(a) $\mathrm{B}$

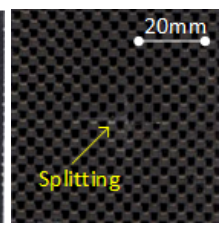

(b) $\mathrm{C}$

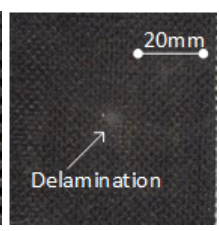

(c) $\mathrm{BCs}$

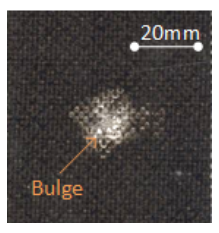

(d) $\mathrm{BCa}$
Fig. 2. Photographs of the impact regions: back side.
Fig 2 shows the photographs of the impact regions from the rear side. CFRP specimen shows penetration of the dart through the thickness with splitting due to their brittle nature (Fig. 2b). On the contrary, BFRP specimen shows delamination without back surface splitting. BCs and BCa show an intermediate damage pattern with respect to BFRP and CFRP specimens. In particular, a bulge is present on the surface of $\mathrm{BCa}$. At least, this is true after a visual inspection.

\section{Methodology}

\section{A. Optical Excitation Thermography}

Optical excitation thermography includes pulsed thermography (PT) and lock-in thermography (LT) experimental set-ups among others.

In PT, photographic flashes or high-energy lamps are used to generate a heat pulse on the specimen surface. The heat transmits itself through the specimen, by diffusion, and then returns to the specimen surface. As time elapses, the surface temperature decreases uniformly for a specimen without internal flaws. On the contrary, subsurface discontinuities can be thought of as resistances to heat flow that change the diffusion rate and produce abnormal temperature patterns at the surface. If these patterns are large enough, then they can be detected with an IR camera and only a few $\mathrm{mK}$ as $\triangle \mathrm{T}$ is needed for the detection of thermal imprints using modern thermographic imaging equipment [20].

The Fourier equation for the propagation of a Dirac heat pulse in a semi-infinite isotropic solid by conduction is [21]:

$$
T(z, t)=T_{0}+\frac{Q}{\sqrt{k \rho c_{p} \pi t}} e\left(-\frac{z^{2}}{4 \alpha t}\right)
$$

where, $Q\left[J / m^{2}\right]$ is the energy absorbed by the surface and $T_{0}[K]$ is the initial temperature.

The Dirac heat pulse consists of periodic waves at all frequencies and amplitudes. A photographic flash provides an approximately square-shaped heat pulse, which can be considered as a convenient approximation. Therefore, the signal can be decomposed by periodic waves at several frequencies. The shorter the pulse, the broader the range of frequencies.

LT, derived from photothermal radiometry, is also known as modulated thermography. In the LT configuration, the absorption of modulated optical heating leads to a temperature modulation, which transmits itself through the specimen as a thermal wave. When the thermal wave is reflected by the defect boundary, the superposition to the original thermal wave will lead to the transformation of the response signal amplitude and phase on the surface. These signals are simultaneously recorded by the IR camera. Sinusoidal waves are typically used in LT, which has the following advantages: 1) frequency and shape of the response are preserved, and 2) only the amplitude and phase delay of the wave may change [20].

Fourier's law for a periodic thermal wave propagating through a semi-infinite homogeneous material can be expressed as [22]:

$$
T(z, t)=T_{0} e^{(-z / \mu)} \cos \left(\frac{2 \pi \cdot z}{\lambda}-\omega t\right)
$$


where, $T_{0}\left[{ }^{\circ} \mathrm{C}\right]$ is the initial temperature change, $\omega=$ $2 \pi f[\mathrm{rad} / \mathrm{s}]$ is the modulation frequency, $f[H z]$ is the frequency, $\lambda[m]$ is the thermal wavelength: $\lambda=2 \pi \mu$, and $\mu[m]$ is the thermal diffusion length, which determines the rate of decay of the thermal wave as it penetrates through a material:

$$
\mu=\sqrt{\frac{2 \cdot \alpha}{\omega}}=\sqrt{\frac{\alpha}{\pi \cdot f}}
$$

The amplitude and the phase delay can be calculated from the Fourier transform as detailed in section III-C1.

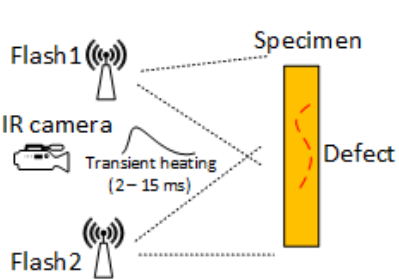

(a)

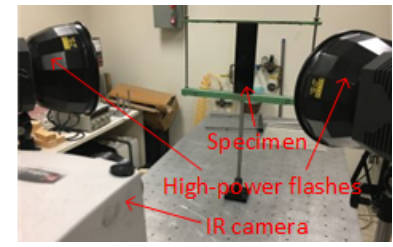

(c)

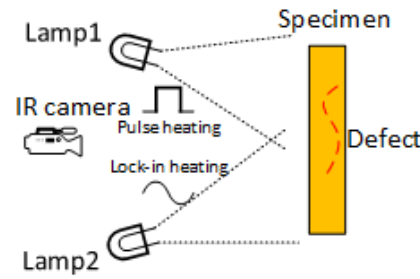

(b)

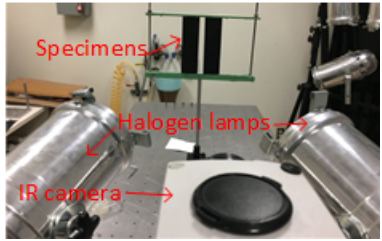

(d)
Fig. 3. Optical excitation thermography configurations: (a) schematic set-up for PT using flashes, (b) schematic set-up for LT and PT using lamps, (c) experimental set-up for PT using flashes, (d) experimental set-up for LT and PT using lamps.

Fig. 3a shows the schematic set-up for photographic flash modality, while the modulated lamp modality is shown in Fig. $3 \mathrm{~b}$. Figs. $3 \mathrm{c}$ and $3 \mathrm{~d}$ show the real experimental set-ups. Two Balcar FX 60 (6.4 KJ, 2 ms duration) producing optical flashes were used in this configuration, while two 'OMNLUX PAR64' (1000 W, $5 \mathrm{~s}$ duration) halogen lamps were employed for lockin thermography inspection. A mid-wave IR camera 'Flir Phoenix' with the frame rate of $\sim 55 \mathrm{fps}$ and the NETD of $25 \mathrm{mK}$ was used. The cooling times were set at $10 \mathrm{~s}$ and 20 $\mathrm{s}$ for flashes and halogen lamps modalities, respectively. The camera spatial resolution is $640 \times 512$ pixels $(25 \mu m \times 25 \mu m$ of detector size) and a $50 \mathrm{~mm}$ lens was employed to provide a field of view (FOV) of $18.2^{\circ}$ (horizontal) x $14.6^{\circ}$ (vertical). Hence, from a distance of $50 \mathrm{~cm}$ between the camera and the specimens, the FOV in $\mathrm{cm}$ is 16.0 (horizontal) x 12.8 (vertical), which corresponds to 40 pixels $/ \mathrm{cm}$.

\section{B. Mechanical Excitation Thermography}

Vibrothermography (VT) uses mechanical waves to stimulate internal defects without heating the surface as in optical excitation thermography. Photothermal radiometry can be considered as the predecessor of optical thermography; VT should be the successor of optoacoustics or photoacoustics phenomena in which microphones or piezoceramics in contact with the specimen and a lock-in amplifier were used to detect the thermal wave signature from a defect. In VT, ultrasonic waves travel through a specimen, in which an internal defect results in a complex combination of absorption, scattering, beam spreading and dispersion of the waves. The waves generate heat which subsequently travels by conduction in all directions [20]. An IR camera faces the surfaces of the specimen to capture the defect signature at the surface.

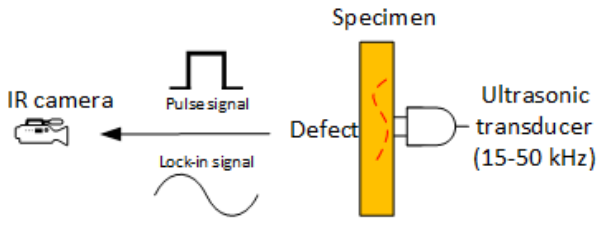

(a)

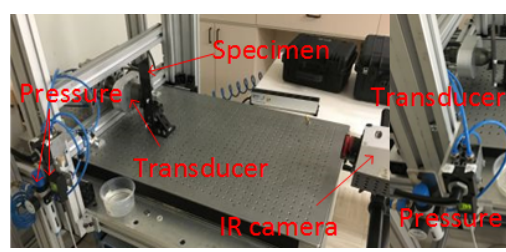

(b)

Fig. 4. VT configuration, (a) schematic set-up, (b) experimental set-up.

Fig. 4 shows the VT set-up. The previous IR camera with the same frame rate equal to $\sim 55 \mathrm{fps}$ was used. The transducer was pressed (200 Pa) against the specimen and two periods of $0.2 \mathrm{~Hz}(10 \mathrm{~s})$ lock-in ultrasonic waves were delivered.

\section{Image Processing and Analysis}

In this paper, recursive median filter (RMF) and cold image subtraction (CIS) [23] were performed prior to the application of pulsed phase thermography (PPT), principal component thermography (PCT) and the partial least squares thermography (PLST) technique in PT regime, while RMF was applied prior to the application of phase transform in LT.

1) PPT: Discrete Fourier transform (DFT) can be used to extract amplitude and phase information from LT and PT data. The most important characteristic of phase and amplitude transform is that they provide the possibility to obtain quantitative results in a straightforward manner. A relationship exists between the depth $z$ of a defect and the thermal diffusion length $\mu$. Physico-mathematical expressions have been proposed, such as [24]:

$$
z=C_{1} \cdot \mu=C_{1} \cdot \sqrt{\frac{\alpha}{\pi \cdot f_{b}}}
$$

where, $f_{b}$ is known as the blind frequency - the frequency at which a given defect has enough (phase or amplitude) contrast to be detected, while $C_{1}$ is calculated after a series of experiments.

It has been observed that $C_{1} \approx 1$ for amplitude transform, while reported values for phase transform are in the range of 1.5 to 2 , with $C_{1}=1.82$ typically adopted for similar cases such as the present [25]. Therefore, the phase is more useful than the amplitude because it can retrieve the deeper information. 
In addition, the phase $\phi_{n}$ is less affected than raw and amplitude data by environmental reflections, emissivity variations, non-uniform heating, surface geometry, and orientation. These phase characteristics are very attractive not only for qualitative inspections but also for quantitative characterization of materials [20]. More information regarding PPT can be found in [26].

2) PLST: Based on a statistical correlation method known as partial least squares regression (PLSR), PLST computes loading $P$ and score $T$ vectors that are correlated to the predicted block $Y$ (as in maximum redundancy analysis), while describing a large amount of the variation in the predictor matrix $X$ (as in principal component regression). The matrix $X$ corresponds to the surface temperature matrix obtained during the PT inspection, while $Y$ is defined by the observation time during which the thermal images were captured. The PLS model is achieved by decomposing $X$ and $Y$ into a combination of loadings $P$ and $Q$ (formed by orthogonal vectors), scores $T$ and $U$ (the projections of the loading vectors associated with the singular values) and residuals $E$ and $F$. Mathematically, the PLS model is expressed as:

$$
\begin{aligned}
& X=T P^{\prime}+E \\
& Y=U Q^{\prime}+F
\end{aligned}
$$

In order to perform the decomposition of the thermal sequence matrix (which in fact is a regression), it is necessary to select the appropriate number of PLS components. More information regarding PLST can be found in [27].

3) PCT: This technique extracts both the image features and reduces undesirable signals. It relies on singular value decomposition, which is a factorization method to extract spatial and temporal data from a matrix in a compact manner by projecting original data onto a system of orthogonal components known as empirical orthogonal functions (EOF). The first EOF will represent the most important characteristic variability of the data; the second EOF will contain the second most important variability, and so on. Usually, original data can be represented with only a few EOFs. Typically, an infrared sequence of 1000 images can be replaced by 10 or fewer EOF. For a large amount of frames and pixels, more EOFs are preferred. More information regarding the PCT technique can be found in [28].

4) SNR: This data analysis method is proposed herein as a means to evaluate and compare the sensitivity of the applied processing techniques. The SNR is expressed in decibels $(\mathrm{dB})$ following the $20 \mathrm{log}$ rule often used in imaging applications, as follows:

$$
S N R=20 \cdot \log _{10}\left|\frac{S_{d}-S_{a}}{\sigma_{S a}}\right|
$$

where $S_{d}$ represents the intensity of signals, $S_{a}$ represents the intensity of sound areas, and $\sigma_{S a}$ is the standard deviation representing noise variability.

SNR values provide important information about the sensitivity of each technique when comparing the detectability of similar features or defects.

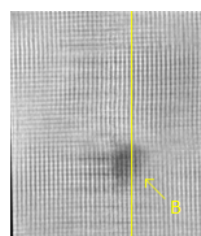

(a) $\mathrm{B}: 0.6 \mathrm{~mm}$

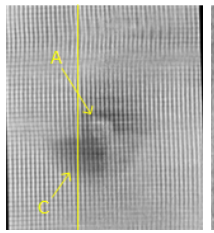

(e) $\mathrm{B}: 0.8 \mathrm{~mm}$

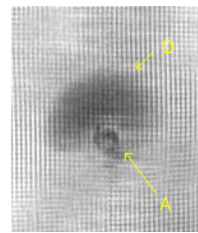

(i) $\mathrm{B}: 1.2 \mathrm{~mm}$

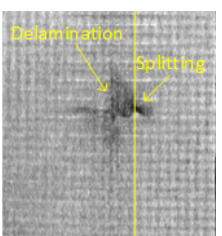

(b) $\mathrm{C}: 0.5 \mathrm{~mm}$

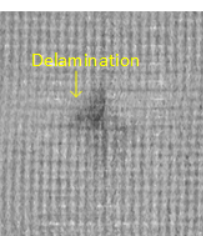

(f) $\mathrm{C}: 1.7 \mathrm{~mm}$

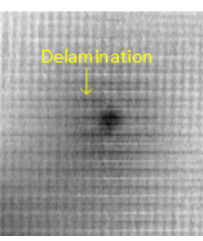

(j) C: $3.3 \mathrm{~mm}$

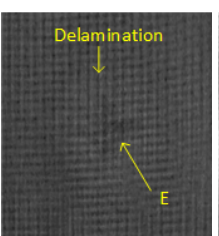

(c) $\mathrm{BCa}: 0.8 \mathrm{~mm}$

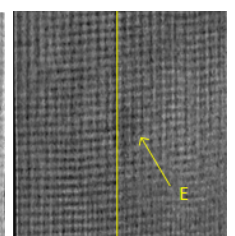

(g) BCs: $1.7 \mathrm{~mm}$

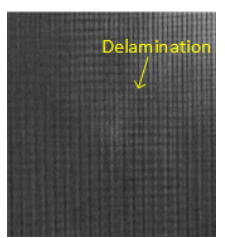

(k) BCs: $2.7 \mathrm{~mm}$

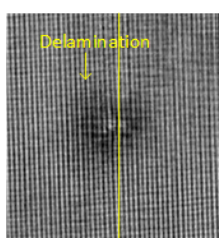

(d) BCa: $0.8 \mathrm{~mm}$
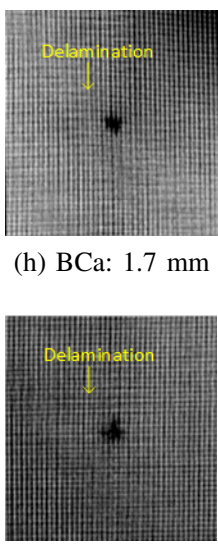

(1) BCa: $2.7 \mathrm{~mm}$ (h) $\mathrm{BCa}: 1.7 \mathrm{~mm}$

Fig. 5. CT slices of the four specimens at different depths as indicated.

\section{EXPERIMENTAL RESULTS AND ANALYSIS}

Fig. 5 shows the $100 \mu$ m-resolution CT slices, which were used to validate the thermographic results. In BFRP specimen, Region B is the clearest at a depth of $0.6 \mathrm{~mm}$ (Fig. 5a), while region $\mathrm{C}$ appears at a depth of $0.8 \mathrm{~mm}$ (Fig. 5e). Regions $\mathrm{B}$ and $\mathrm{C}$ disappear at a depth of $1.2 \mathrm{~mm}$ (Fig. 5i), while region $\mathrm{D}$ appears from this depth. Side-view slices Figs. 6a and $6 \mathrm{~b}$ show the clearer positions of regions $\mathrm{B}, \mathrm{C}$ and $\mathrm{D}$. In the CFRP specimen, specific delamination appears at the depths of $0.5 \mathrm{~mm}$ and $1.7 \mathrm{~mm}$. Fig. $6 \mathrm{c}$ shows the side-view slice linked to the cutting line in Fig. 5b. In the slices of the hybrid specimens, BCs shows a decreasing delamination extension along with the depth. However, it shows a sharply decreasing delaminated area at a depth of $1.7 \mathrm{~mm}$ (Fig. $5 \mathrm{~g}$ ), which is located at the middle depth (carbon fiber fabric core). The delamination positions in BCs are deeper than those in $\mathrm{BCa}$ as shown in Figs. 6d and 6e.

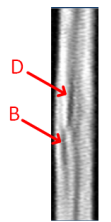

(a) B

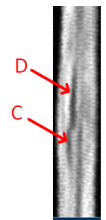

(b) $\mathrm{B}$

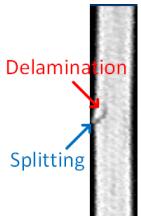

(c) $\mathrm{C}$

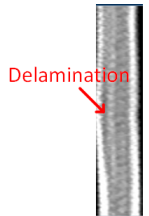

(d) BCs

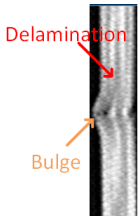

(e) $\mathrm{BCa}$
Fig. 6. CT slices (side-view) linked to the cutting lines (marked in yellow) in : (a) Fig. 5a, (b) Fig. 5e, (c), Fig. 5b, (d) Fig. 5g, (e) Fig. 5d.

Fig. 7 shows the PCT results from the PT configuration using the flashes set-up. The BFRP specimen has the largest delaminated area among all of the specimens (Fig. 7a), while the CFRP specimen has the smallest delaminated area. The BCs specimen (Fig. 7c) possesses a similar behaviour to that of 


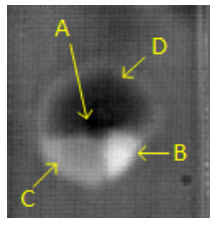

(a) $\mathrm{B}$

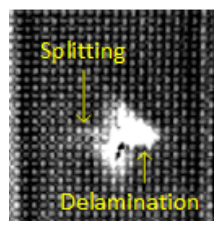

(b) $\mathrm{C}$

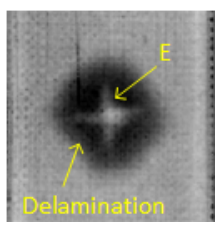

(c) $\mathrm{BCs}$

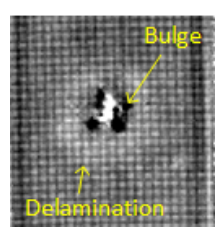

(d) $\mathrm{BCa}$
Fig. 7. PCT results of PT: EOF 4 in all cases.

the BFRP specimen. The BCa specimen with a bulge (Fig. 7d) shows a similar delaminated area as that of the BCs specimen, although slightly less extensive. This phenomenon coincides with the CT results presented in Fig. 5. The BCs specimen

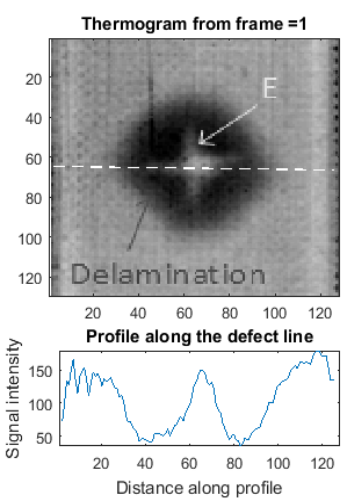

(a) BCs: linked to Fig. $7 \mathrm{c}$

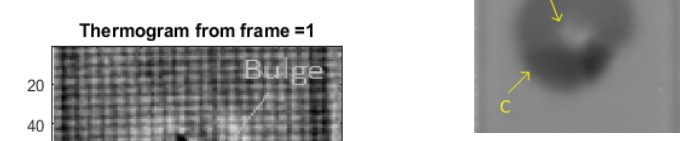

(e) B: 2nd

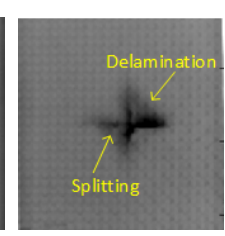

(b) $\mathrm{C}: 1 \mathrm{st}$

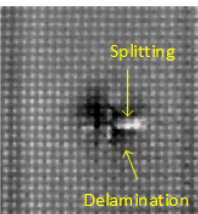

(f) C: 2nd

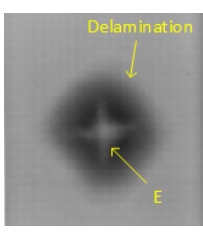

(c) BCs: $1 \mathrm{st}$

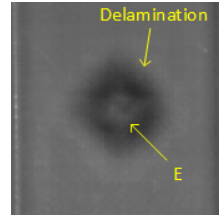

(g) BCs: 2nd

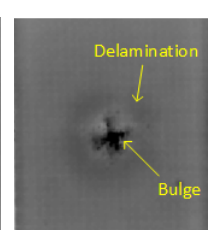

(d) BCa: 1st

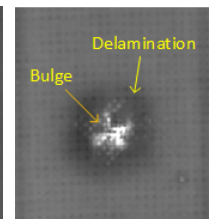

(h) BCa: 2nd

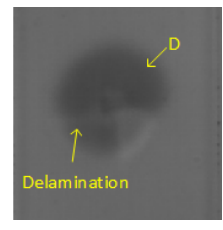

(i) $\mathrm{B}: 3 \mathrm{rd}$

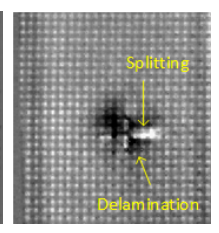

(j) C: 3rd

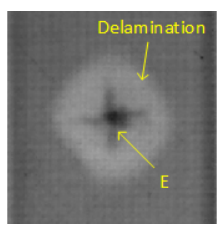

(k) BCs: 3rd

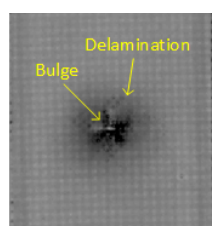

(1) BCa: 3rd
Fig. 10. PLST results of the four specimens for different loadings as indicated.

Fig. 8. SNR analysis for the BCs and BCa specimens in PCT (white lines).

shows a larger SNR value than the BCa specimen as shown in Fig. 8, which is linked to Figs. 7c and 7d. This indicates that the position of delamination in $\mathrm{BCa}$ specimen is deeper than that in the BCs specimen, which can be validated by the CT results. Of particular interest, cross-shaped defects A and $\mathrm{E}$ were detected in the BFRP and BCs specimens. In addition, regions $\mathrm{B}, \mathrm{C}$ and $\mathrm{D}$ have different thermal signatures, as shown in Fig. 7a. This indicates that B, C and D are located at different depths, which can also be validated by the $\mathrm{CT}$ results.

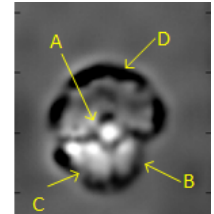

(a) $\mathrm{B}$

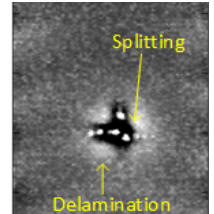

(b) $\mathrm{C}$

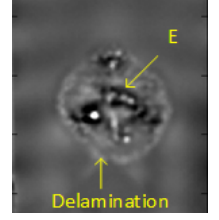

(c) BCs

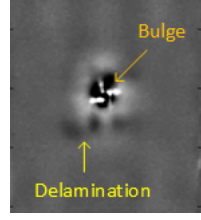

(d) $\mathrm{BCa}$

Fig. 9. PCT results of VT: (a) EOF 3, (b) EOF 3, (c) EOF 4, (d) EOF 3.

Fig. 9 shows the PCT results of VT. BFRP and BCs specimens show clearer delaminated areas than CFRP and $\mathrm{BCa}$ specimens. Cross-shaped defects A and $\mathrm{E}$ were inspected more clearly in Figs. 9a and 9c. Contrary to the PT result (Fig. 7a), regions $\mathrm{B}, \mathrm{C}$ and $\mathrm{D}$ show similar contrast. This seems to be due to the fact that heat is generated internally, at the defect interfaces, contrary to optical stimulation in which heat travels from the surface to the defect providing additional information

PLST, which can provide images along with the increase of depth, was used to process the PT data. Fig. 10 shows the PLST results. Halogen lamps were used since they can detect deeper depths than flashes. Fig. 11a shows the bar graphs of

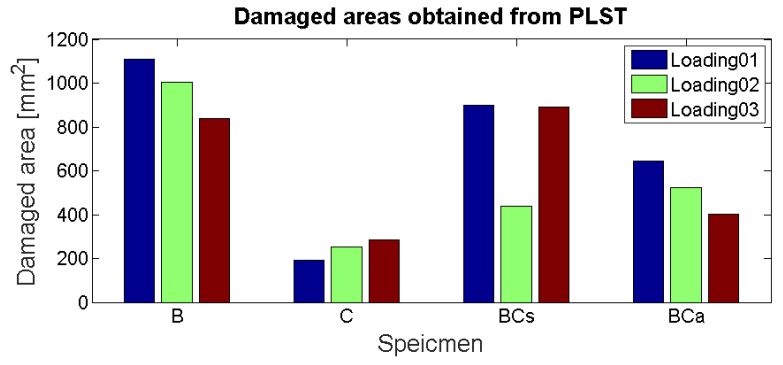

(a) Damaged areas: PLST

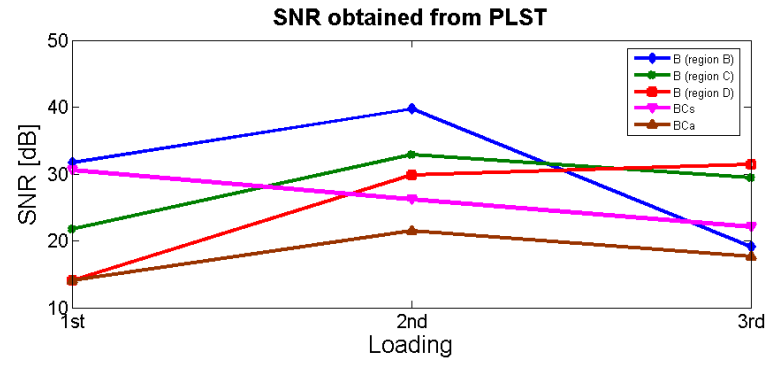

(b) SNR curves: PLST

Fig. 11. Damaged areas and SNR curves obtained from PLST.

damaged areas obtained from PLST. The delaminated area in the BFRP specimen decreases along with the increase of depth. 
This result is reasonable, since BFRP has better toughness. The absorbed energy $E_{a}$ created the delamination, but not splitting. Usually the delaminations are located near the back surface [29]. Of interest, region B is not present in Figs. 10e and 10i. This indicates that region B is the nearest to the back surface and region $\mathrm{D}$ is located deepest (near the impacted surface). The delaminated area in the $\mathrm{BCa}$ specimen decreases along with the increase of depth. However, BCs shows a different reaction after the impact in the middle of the depth axis (Fig. $10 \mathrm{~g}$ ). This result should be viewed taking into account that the sandwich-like cores consist of carbon fiber fabrics.

Fig. 11b shows the SNR values obtained from PLST. The regions $B$ and $C$ show a peak value both at Loading 02 , which provides an indication of the corresponding depth linked to Loading 2 in the BFRP specimen. The depth linked to Loading 2 in CFRP is $\sim 0.7 \mathrm{~mm}$ where the regions $\mathrm{B}$ and $\mathrm{C}$ both show the clearest contrast in the CT results. The SNR value of region $\mathrm{D}$ increase along with the depth, which indicates that the depth linked to Loading 3 in BFRP is $\geq 1.2 \mathrm{~mm}$ linked to the CT slice Fig. 5i. The SNR value of delamination in BCs specimen decreases along with the depth, while the SNR value of delamination in $\mathrm{BCa}$ specimen shows a peak value at Loading 2. This phenomenon indicates that the position of delamination in $\mathrm{BCa}$ is deeper than the one in $\mathrm{BCs}$, which can be validated the $\mathrm{CT}$ results.

The PLST technique can link the loadings with the increase of the depths and the depths linked to Loadings can be estimated by SNR analysis. However, CT slices are needed for depth estimation and it cannot provide depth values by thermal physics. This explains why PPT and LT were also used in this paper; indeed, they can take advantage of phase transform to estimate the depth of defects. Specifically, the thermal diffusivity $\alpha$ is a parameter of particular interest for calculating the depth by phase transform according to Eq. 4, and can be expressed as follows according to Eq. 1:

$$
\alpha=\frac{k}{\rho c_{p}}
$$

where, $c_{p}[J / k g K]$ is the specific heat at constant pressure, $\rho\left[\mathrm{kg} / \mathrm{m}^{3}\right]$ is the density, and $k[W / m K]$ is the thermal conductivity.

For composite materials, $\alpha$ can be calculated by [30]:

$$
\alpha_{\text {composite }}=\sum_{1}^{N}\left(W_{f} \cdot \alpha_{\text {material }}\right)_{N}
$$

where, $N$ is the number of constituent materials in the composite, and $W_{f}$ is the fiber weight fraction.

Eq. 9 is linked to the fact that the $\alpha$ parameter is composed of physical quantities, but not of pseudo-physical quantities, in a similar manner as the $C_{p}$ parameter. Therefore, taking into account the Buckingham theorem, it is possible to sum each layer for its volume fraction and mass fraction, respectively. This enables the final $\alpha$ and volume parameters to be obtained. Table I shows the thermal diffusivity $\alpha$ calculated according to Eq. 9, while Table II shows the relationship between modulated frequencies $f_{b}$ and depth $z$ according to Eq. 4.

Fig. 12 shows the PPT phase results. It is possible to see how PPT displays a phase image similar to those obtained
TABLE I

CALCUlATED THERMAL DifFusivity $\alpha$.

\begin{tabular}{c|ccc}
\hline Specimen & B & C & B-C \\
\hline Thermal diffusivity $\alpha\left[10^{-7} \mathrm{~m}^{2} / \mathrm{s}\right]$ & 0.88 & 2.08 & 1.43 \\
\hline
\end{tabular}

TABLE II

RELATIONSHIP BETWEEN MODULATED FREQUENCY $f_{b}$ AND DEPTH $z$.

\begin{tabular}{lc|cccccc}
\hline Modulated frequency & $f_{b}[\mathrm{~Hz}]$ & 0.2 & 0.1 & 0.065 & 0.05 & 0.02 & 0.01 \\
\hline \multirow{3}{*}{ Depth $z[\mathrm{~mm}]$} & $\mathrm{B}$ & 0.68 & 0.96 & 1.19 & 1.36 & 2.15 & 3.05 \\
& $\mathrm{C}$ & 1.05 & 1.48 & 1.84 & 2.09 & 3.31 & 4.68 \\
& $\mathrm{~B}-\mathrm{C}$ & 0.87 & 1.23 & 1.52 & 1.74 & 2.75 & 3.88 \\
\hline
\end{tabular}

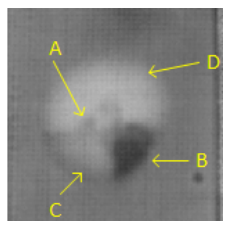

(a) B: $0.68 \mathrm{~mm}$

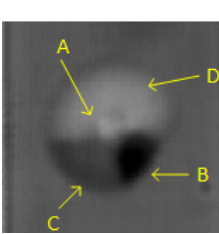

(e) B: $0.96 \mathrm{~mm}$

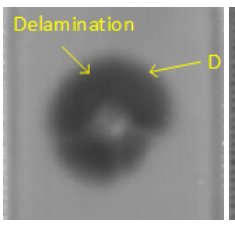

(i) B: $1.19 \mathrm{~mm}$

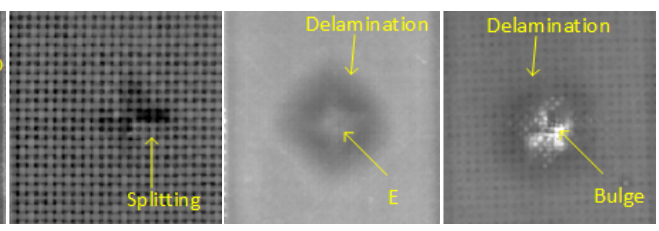

$\begin{array}{lll}\text { (b) } \mathrm{C}: 1.05 \mathrm{~mm} & \text { (c) BCs: } 0.87 \mathrm{~mm} \text { (d) BCa: } 0.87 \mathrm{~mm}\end{array}$

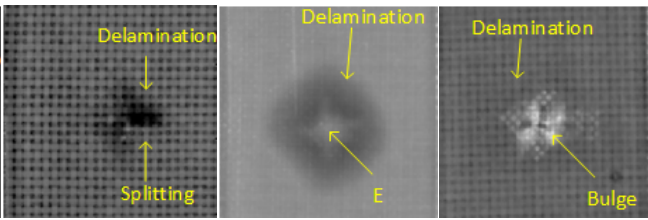

(f) C: $1.48 \mathrm{~mm} \quad$ (g) BCs: $1.23 \mathrm{~mm}$ (h) BCa: $1.23 \mathrm{~mm}$

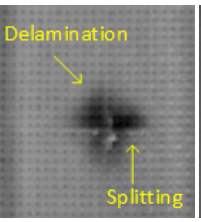

(j) C: $1.84 \mathrm{~mm}$ (k) BCs: $1.52 \mathrm{~mm}$ (l) BCa: $1.52 \mathrm{~mm}$
Fig. 12. PPT phase results with depths retrieval.

using the PLST image processing (images along with the depth). In the BFRP specimen, region B has the greatest contrast at the depth of $0.68 \mathrm{~mm}$ and becomes blurry beyond a depth of $1.19 \mathrm{~mm}$ (Fig. 12i), while region $\mathrm{C}$ is better detected at the depth of $0.96 \mathrm{~mm}$ (Fig. 12e). Region D appears between the depths of $0.68 \mathrm{~mm}$ and $1.19 \mathrm{~mm}$. These defect discrimination features provide a clear indication of the defects depths. In the CFRP specimen, specific delaminated areas appear at the depths of $1.05 \mathrm{~mm}$ and $1.48 \mathrm{~mm}$. An increasing damage is shown along with the depth. Fig. 13 presents phase images obtained from LT that show similar damaged areas as the PT phase images in Fig. 12. Additionally, lower modulation frequencies were also employed in LT to probe deeper as seen in the bottom images in Fig. 13.

Fig. 14a shows an estimation of the damaged areas obtained from PPT and LT phase results. In the hybrid specimens, $\mathrm{BCa}$ shows a decrease in the delamination area along with the depth. However, BCs shows a particular reaction after the impact loading at the depth of $1.52 \mathrm{~mm}$, as shown in Fig. $12 \mathrm{k}$. This result coincides with the corresponding PLST results (Fig. 10g). The SNR analysis in Fig. 15 shows the coincidence more clearly. This phenomenon is caused by the sandwich-like 


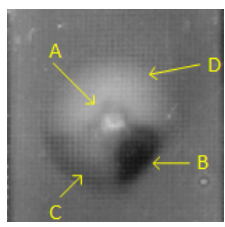

(a) B: $0.68 \mathrm{~mm}$

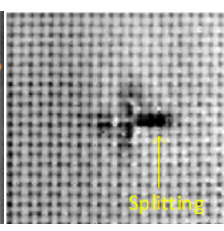

(b) $\mathrm{C}: 1.05 \mathrm{~mm}$

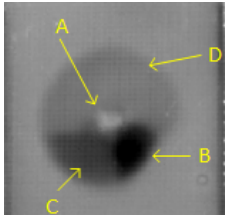

(e) B: $0.96 \mathrm{~mm}$

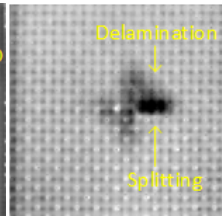

(f) $\mathrm{C}: 1.48 \mathrm{~mm}$

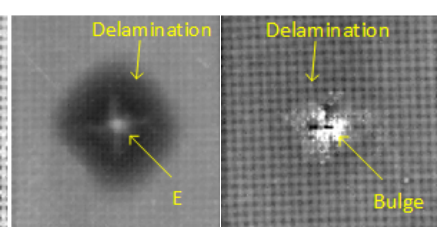

(c) BCs: $0.87 \mathrm{~mm}(\mathrm{~d}) \mathrm{BCa}: 0.87 \mathrm{~mm}$ (i) $\mathrm{B}: 1.36 \mathrm{~mm}$

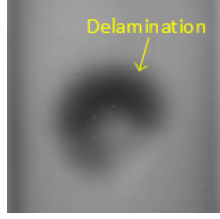

(m) B: $3.05 \mathrm{~mm}$

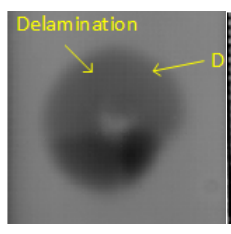

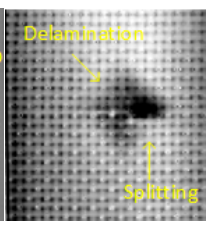

(j) $\mathrm{C}: 2.09 \mathrm{~mm}$

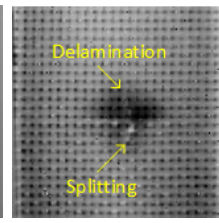

(n) $\mathrm{C}: 3.31 \mathrm{~mm}$

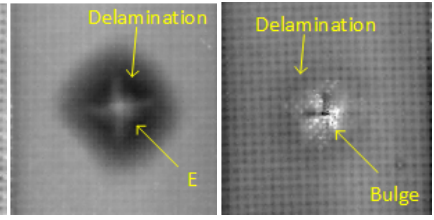

(g) BCs: $1.23 \mathrm{~mm}(\mathrm{~h})$ BCa: $1.23 \mathrm{~mm}$
Fig. 13. LT phase results with depths retrieval.

structure (carbon fiber fabric core).

In the SNR analysis, PPT and LT show similar values and trends (Figs. 14b and 14c). In BFRP, region B shows a peak value at a frequency of $0.1 \mathrm{~Hz}$, while regions $\mathrm{C}$ and $\mathrm{D}$ show a peak value at frequencies of $0.065 \mathrm{~Hz}$ and $0.05 \mathrm{~Hz}$ in PPT and $\mathrm{LT}$, respectively. This phenomenon indicates that regions $\mathrm{C}$ and $\mathrm{D}$ are at a deeper depth than region $\mathrm{B}$, which can be validated by the CT results. BCs shows a peak SNR value at the depths of $1.52 \mathrm{~mm}$ and $1.74 \mathrm{~mm}$ in PPT and LT, respectively. This may link to the sandwich-like structure (carbon fiber fabric core) which is in agreement with the analysis of damaged areas. $\mathrm{BCa}$ also shows a peak SNR value at the depths of $1.52 \mathrm{~mm}$ and $1.74 \mathrm{~mm}$ in PT. This may indicate that the depth of delamination in $\mathrm{BCa}$ corresponds to the frequency of maximum SNR, which is consistent with the PLST and CT results.

PPT and LT show similar SNR trends. However, larger differential values may exist when defects are deeper (e.g., region $\mathrm{D}$ in BFRP in 14b) or when intra-laminar defects play a main role (e.g., BCa in Fig. 14c). Therefore, the SNR analysis for CFRP is more complex since the damage is mostly intra-laminar, which results in the inaccuracy of SNR analysis compared with the other specimens whose damages are mostly inter-laminar. Therefore, the SNR analysis for CFRP is not discussed in this paper.

Fig. 16 shows the maximum damaged areas and SNR values obtained from different thermographic techniques. Although

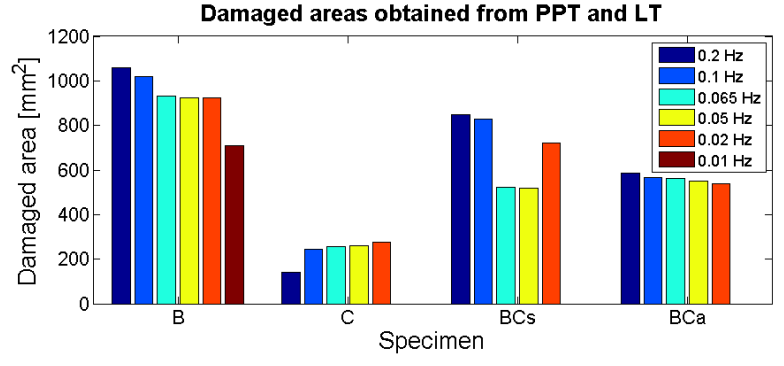

(a) Damaged areas: PPT and LT

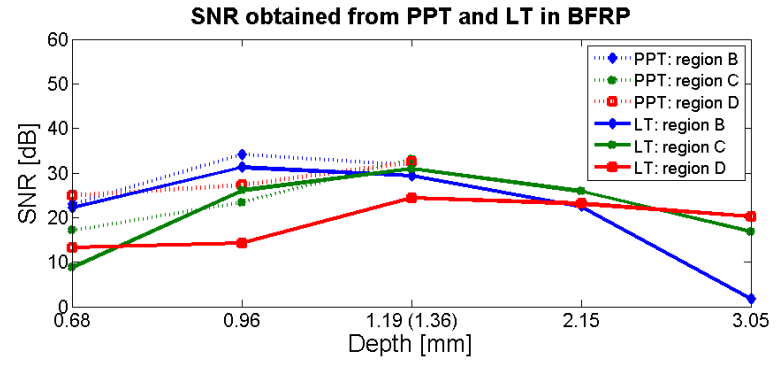

(b) SNR curves in BFRP

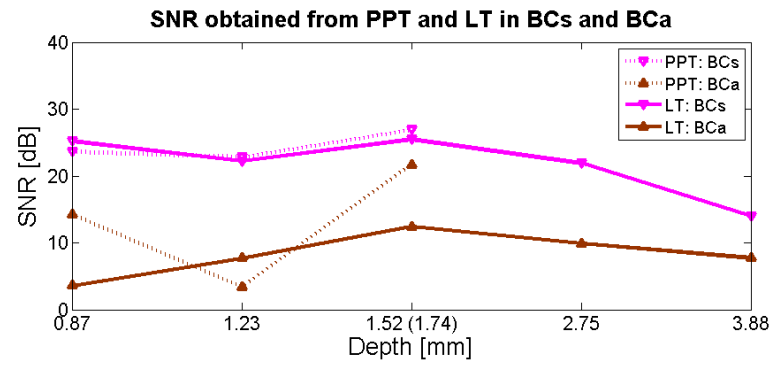

(c) SNR curves in BCs and $\mathrm{BCa}$

Fig. 14. Damaged areas and SNR curves obtained from PPT and LT.

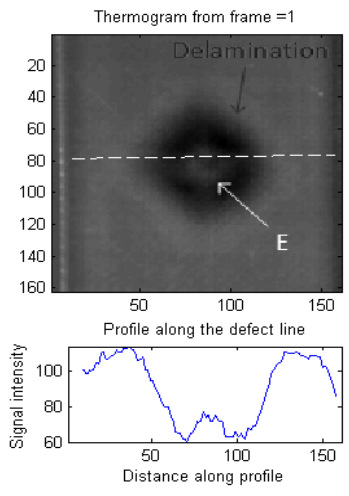

(a) PLST: linked to Fig. $10 \mathrm{~g}$

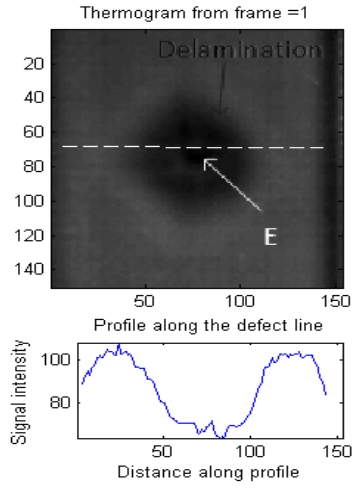

(b) PPT: linked to Fig. $12 \mathrm{k}$
Fig. 15. SNR analysis for the BCs specimen in PLST and PPT (white lines).

the largest damaged areas were observed with the PCT technique (with the exception of BCS), its SNR values are lower than those calculated for PLST, PPT and LT. PLST can detect larger damaged areas than PPT and LT. Figs. 13o and 13p show less information because $3.88 \mathrm{~mm}$ is beyond the depth for these types of specimens. VT is more complex than optical excitation thermography since the excitation occurs inside the specimen. BCa shows complex SNR values for the analysis 


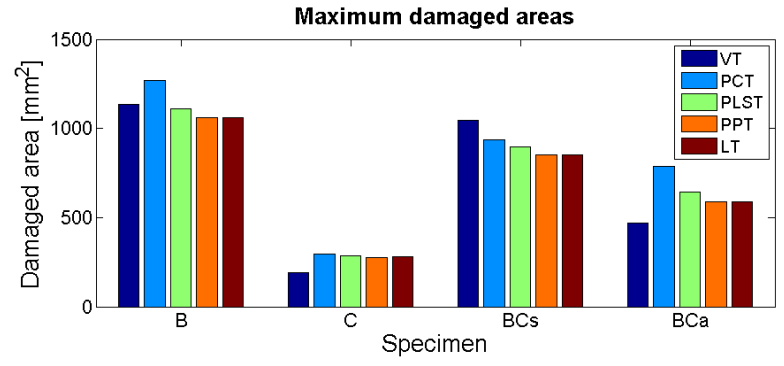

(a) Maximum damaged areas

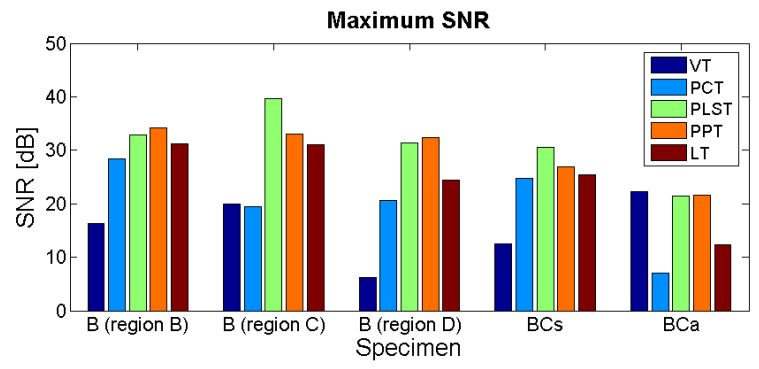

(b) Maximum SNR values

Fig. 16. Maximum damaged areas and SNR values.

since the structure is intercalated. A finite element analysis should contribute to the understanding of thermal behavior of basalt-carbon hybrid laminates [31].

\section{CONCLUSIONS}

Both optical and mechanical excitation thermography are useful imaging techniques for impact damage in BFRP, CFRP and basalt-carbon fiber hybrid composites. If compared to the $100 \mu \mathrm{m}$-resolution $\mathrm{CT}$ technique which was used to validate the thermographic results, IRT has the abilities to provide clear images and additional information. The depth information can be calculated by thermal physics in PPT and LT. SNR can be a means of quantitatively comparing the thermographic results. Of particular interest, the depth information linked to Loadings in PLST was estimated for the first time. However, this method needs a reference technique such as CT.

Absorbed energy $E_{a}$ was more easily converted into delamination in BFRP thanks to its lower interlaminar shear strength. On the contrary, $E_{a}$ created back surface splitting in CFRP due to its inherent limited ductility. Hybridization with basalt fibers improved the toughness of carbon-based laminates to a different extent depending on the stacking sequence. The basalt-carbon fiber hybrid specimen (BCs) with sandwichlike structure shows a pronounced delaminated area similar to that observed in the BFRP specimen, while the basalt-carbon fiber hybrid specimen $(\mathrm{BCa})$ with alternately stacked structure shows characteristics much more similar to those of the CFRP specimen. Summarizing, a more extensive delaminated area was detected in the BCs specimen than in the $\mathrm{BCa}$ specimen. This is because delaminations are probably more numerous in the $\mathrm{BCa}$ specimen, but less extensive due to the multiple basalt-carbon interfaces. In addition, the delamination in $\mathrm{BCa}$ is at a deeper location than that in BCs.

This study provides a reference for taking advantage of different basalt-carbon fiber hybrid structures for specific in- dustrial applications. In particular, it confirmed the possibility of detecting damage at different depths. This is of particular interest with a view to correlating the damage and its extension with the residual properties of composite laminates. A simulation using the finite element method (FEM) may contribute to the further understanding of sandwich-like and alternating stacked structural characteristics subjected to known velocities of impact [31].

\section{REFERENCES}

[1] W.-C. Li and D.-M. Tsai, "Defect inspection in low-contrast lcd images using hough transform-based nonstationary line detection," IEEE Transactions on industrial informatics, vol. 7, no. 1, pp. 136-147, 2011.

[2] B. Gao, W. L. Woo, G. Y. Tian, and H. Zhang, "Unsupervised diagnostic and monitoring of defects using waveguide imaging with adaptive sparse representation," IEEE Transactions on Industrial Informatics, vol. 12, no. 1, pp. 405-416, 2016.

[3] B. Gao, W. L. Woo, Y. He, and G. Y. Tian, "Unsupervised sparse pattern diagnostic of defects with inductive thermography imaging system," IEEE Transactions on Industrial Informatics, vol. 12, no. 1, pp. 371$383,2016$.

[4] K. Ahi, S. Shahbazmohamadi, and N. Asadizanjani, "Quality control and authentication of packaged integrated circuits using enhanced-spatialresolution terahertz time-domain spectroscopy and imaging," Optics and Lasers in Engineering, vol. in press, 2017.

[5] X. Bai, Y. Fang, W. Lin, L. Wang, and B.-F. Ju, "Saliency-based defect detection in industrial images by using phase spectrum," IEEE Transactions on Industrial Informatics, vol. 10, no. 4, pp. 2135-2145, 2014.

[6] G. Acciani, G. Brunetti, and G. Fornarelli, "Application of neural networks in optical inspection and classification of solder joints in surface mount technology," IEEE Transactions on industrial informatics, vol. 2, no. 3, pp. 200-209, 2006.

[7] D.-M. Tsai, I.-Y. Chiang, and Y.-H. Tsai, "A shift-tolerant dissimilarity measure for surface defect detection," IEEE Transactions on Industrial Informatics, vol. 8, no. 1, pp. 128-137, 2012.

[8] C. Meola, S. Boccardi, G. Carlomagno, N. Boffa, F. Ricci, G. Simeoli, and P. Russo, "Impact damaging of composites through online monitoring and non-destructive evaluation with infrared thermography," NDT \& E International, vol. 85, pp. 34-42, 2017.

[9] Y. Li, Z.-w. Yang, J.-t. Zhu, A.-b. Ming, W. Zhang, and J.-y. Zhang, "Investigation on the damage evolution in the impacted composite material based on active infrared thermography," NDT \& E International, vol. 83, pp. 114-122, 2016.

[10] A. Picón, O. Ghita, P. F. Whelan, and P. M. Iriondo, "Fuzzy spectral and spatial feature integration for classification of nonferrous materials in hyperspectral data," IEEE Transactions on Industrial Informatics, vol. 5, no. 4, pp. 483-494, 2009.

[11] Y. Si, J. Mei, and H. Gao, "Novel approaches to improve robustness, accuracy and rapidity of iris recognition systems," IEEE transactions on industrial informatics, vol. 8, no. 1, pp. 110-117, 2012.

[12] L. Sripragash and M. J. Sundaresan, "A normalization procedure for pulse thermographic nondestructive evaluation," NDT \& E International, vol. 83, pp. 14-23, 2016.

[13] B. Yousefi, S. Sfarra, C. I. Castanedo, and X. P. Maldague, "Comparative analysis on thermal non-destructive testing imagery applying candid covariance-free incremental principal component thermography (ccipct)," Infrared Physics \& Technology, vol. 85, pp. 163-169, 2017.

[14] D.-M. Tsai and J.-Y. Luo, "Mean shift-based defect detection in multicrystalline solar wafer surfaces," IEEE Transactions on Industrial Informatics, vol. 7, no. 1, pp. 125-135, 2011.

[15] D. P. Almond, S. L. Angioni, and S. G. Pickering, "Thermographic nde advisory and guidance system," NDT \& E International, vol. 83, pp. 134-142, 2016.

[16] M. Aktaş, C. Atas, B. M. İçten, and R. Karakuzu, "An experimental investigation of the impact response of composite laminates," Composite Structures, vol. 87, no. 4, pp. 307-313, 2009.

[17] T. Deák and T. Czigány, "Chemical composition and mechanical properties of basalt and glass fibers: a comparison," Textile Research Journal, vol. 79, no. 7, pp. 645-651, 2009.

[18] V. Fiore, T. Scalici, G. Di Bella, and A. Valenza, "A review on basalt fibre and its composites," Composites Part B: Engineering, vol. 74, pp. 74-94, 2015. 
[19] F. Sarasini, J. Tirillò, L. Ferrante, M. Valente, T. Valente, L. Lampani, P. Gaudenzi, S. Cioffi, S. Iannace, and L. Sorrentino, "Drop-weight impact behaviour of woven hybrid basalt-carbon/epoxy composites," Composites Part B: Engineering, vol. 59, pp. 204-220, 2014.

[20] C. Ibarra-Castanedo and X. P. Maldague, "Infrared thermography," in Handbook of technical diagnostics, H. Czichos, Ed. Springer Berlin Heidelberg, 2013, pp. 175-220.

[21] B. Bein, J. Gibkes, J. Gu, R. Hüttner, J. Pelzl, D. Balageas, and A. Déom, "Thermal wave characterization of plasma-facing materials by ir radiometry," Journal of nuclear materials, vol. 191, pp. 315-319, 1992.

[22] D. Wu and G. Busse, "Lock-in thermography for nondestructive evaluation of materials," Revue générale de thermique, vol. 37, no. 8, pp. 693-703, 1998.

[23] H. Zhang, L. Yu, U. Hassler, H. Fernandes, M. Genest, F. Robitaille, S. Joncas, W. Holub, Y. Sheng, and X. Maldague, "An experimental and analytical study of micro-laser line thermography on micro-sized flaws in stitched carbon fiber reinforced polymer composites," Composites Science and Technology, vol. 126, pp. 17-26, 2016.

[24] J. W. Cooley and J. W. Tukey, "An algorithm for the machine calculation of complex fourier series," Mathematics of computation, vol. 19, no. 90, pp. 297-301, 1965.

[25] G. Busse, "Nondestructive evaluation of polymer materials," $N D T \& E$ International, vol. 27, no. 5, pp. 253-262, 1994.

[26] X. Maldague and S. Marinetti, "Pulse phase infrared thermography," Journal of applied physics, vol. 79, no. 5, pp. 2694-2698, 1996.

[27] F. Lopez, C. Ibarra-Castanedo, V. de Paulo Nicolau, and X. Maldague, "Optimization of pulsed thermography inspection by partial leastsquares regression," NDT \& E International, vol. 66, pp. 128-138, 2014

[28] N. Rajic, "Principal component thermography for flaw contrast enhancement and flaw depth characterisation in composite structures," Composite Structures, vol. 58, no. 4, pp. 521-528, 2002.

[29] P. Servais, N. Gerlach, J. Habermehl, C. Ibarra-Castanedo, and $\mathrm{X}$. Maldague, "Characterization of manufacturing and maintenance of aerospace composite discontinuities using infrared thermography," Materials Evaluation, vol. 66, no. 9, pp. 955-962, 2008.

[30] A.-b. Cherki, A. Khabbazi, B. Remy, and D. Baillis, "Granular cork content dependence of thermal diffusivity, thermal conductivity and heat capacity of the composite material/granular cork bound with plaster,' Energy Procedia, vol. 42, pp. 83-92, 2013.

[31] S. Sfarra, F. López, F. Sarasini, J. Tirillò, L. Ferrante, S. Perilli, C. Ibarra Castanedo, D. Paoletti, E. J. Barbero, and X. Maldague, "Analysis of damage in hybrid composites subjected to ballistic impacts: an integrated non-destructive approach," in Handbook of Composites from Renewable Materials, Physico-Chemical and Mechanical Characterization, V. K. Thakur, M. K. Thakur, and M. R. Kessler, Eds. John Wiley \& Sons, 2017, pp. 175-210.

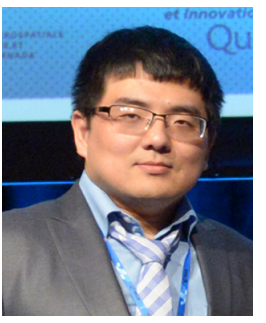

Hai Zhang received the B.Sc. degree in Electronic Science and Technology and the M.Sc. degree in Microelectronics and Solid-State Electronics both from Shenyang University of Technology, Shenyang, China in 2004 and 2008, and the Ph.D. degree in Electrical Engineering from Université Laval, Québec, Canada in 2017, respectively.

From 2012 to 2016, he was a Research Assistant in the Computer Vision and Systems Laboratory (CVSL), Department of Electrical and Computer Engineering, Université Laval, Québec, Canada. In 2015, he was also a Visiting Researcher in Fraunhofer Development Center X-ray Technology (EZRT), Fürth, Germany. In 2017, he was a Visiting Researcher in Fraunhofer Institute for Nondestructive Testing (IZFP), Saarbrücken, Germany and Technical University of Munich, Munich, Germany. From 2017, he began working as a Postdoctoral Research Fellow in the Center for Advanced Diffusion-Wave Technologies (CADIPT), Department of Mechanical and Industrial Engineering, University of Toronto, Toronto, Canada.

He has authored and co-authored more than 30 technical papers in international journals with referees and in international conferences, and has won 2 best paper awards. His research interests include non-destructive evaluation, vision system for industrial inspection, photoacoustic and photothermal imaging, infrared and terahertz spectroscopy.

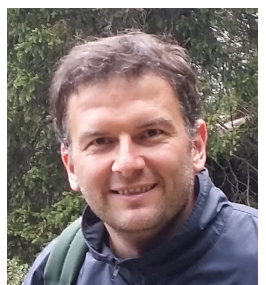

Stefano Sfarra received his degree in Environmental Sciences from University of L'Aquila (Italy) in 2006. In the same University he is, currently, a researcher. In 2011, he also completed a $\mathrm{PhD}$ in Mechanical, Management and Energy Engineering at University of L'Aquila. Since 2012, he is an Adjunct Professor at The School of Restoration The Academy of Fine Arts in L'Aquila, while since March 2012 he is an Invited Researcher in the Computer Vision and Systems Laboratory, Department of Electrical and Computer Engineering, Laval University, Quebec, Canada. As a member of the Las.E.R. Laboratory, he has contributed more than 70 scientific publications based on non-destructive testing (NDT) field, with a special focus on heat transfer. He is also a member of AIPND (Associazione Italiana Prove non Distruttive Monitoraggio e Diagnostica) and AFTI (Associazione della Fisica Tecnica Italiana). His research interests include: NDT\&E, composite materials, cultural heritage, heat transfer, energy saving, numerical simulation and quality control.

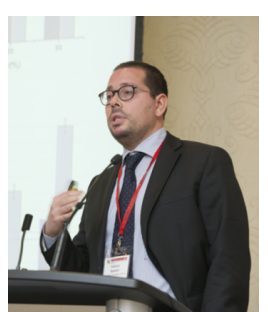

Fabrizio Sarasini received a $\mathrm{Ph}$. D. degree in Materials Engineering from Sapienza University of Rome in 2007. He is currently assistant professor in the Department of Chemical Engineering Materials Environment of the Faculty of Civil and Industrial Engineering of Sapienza University of Rome. His research interests are focused on the assessment of thermo-hygro-mechanical behavior and damage modes of composite materials reinforced with synthetic and natural (both mineral and vegetable) reinforcement, on the response of composite structures to dynamic loads (low and high velocity impacts). He is the author or coauthor of more than 70 peer-reviewed journal papers and 9 invited book chapters.

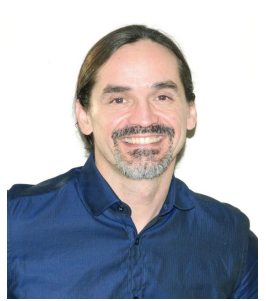

Clemente Ibarra-Castanedo is a professional researcher in the Computer Vision and Systems Laboratory of Laval University in Quebec City, Canada. As a member of the multipolar infrared vision Canada Research Chair (MiViM), he has contributed to many publications in the field of infrared vision. His research interests are in signal processing and image analysis for the nondestructive characterization of materials by active thermography, as well as near and short-wave infrared reflectography/transmittography imaging.

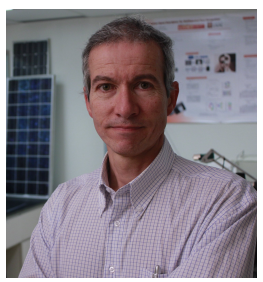

Xavier Maldague is a full professor in the Department of Electrical and Computing Engineering, Université Laval, Québec City, Canada (since 1989, head of the Dept. 2003-2008). He has trained over 50 graduate students (M.Sc. and Ph.D.) and has more than 300 publications. His research interests are infrared thermography, NonDestructive Evaluation (NDE) techniques and vision / digital systems for industrial inspection. He holds a Tier 1 Canada Research Chair in infrared vision. He chairs the Quantitative Infrared Thermography (QIRT) Council. He is a fellow of the Canada Engineering Institute, an Honorary Fellow of the Indian Society of Nondestructive Testing and a fellow of the Alexander von Humbolt Foundation in Germany. Within IEEE Canada, he was CoEditor of the IEEE Canadian Journal of Electrical and Computer Engineering (2003-2007). In 2002-2004 and in 2015-2017, he chaired the IEEE Quebec City Section. In 2004, he was awarded the IEEE J. J. Archambault Award for his dedication to IEEE and his contributions to engineering progress. In 2008-2011, he was the IEEE Canada Publications and Communications Group Chair. 
2017-08-24

\section{Optical and mechanical excitation}

thermography for impact response in

basalt-carbon hybrid fiber-reinforced

composite laminates

Zhang, Hai

IEEE

Zhang H, Sfarra S, Sarasini F, Perilli S, Duan Y, Fernandes H, Peeters J, Avdelidis NP, Ibarra-Castanedo C, Maldague XPV, Optical and mechanical excitation thermography for impact response in basalt-carbon hybrid fiber-reinforced composite laminates, IEEE

Transactions on Industrial Informatics, 2017, Volume 14, Issue 2, pp514-522

http://dx.doi.org/10.1109/TII.2017.2744179

Downloaded from Cranfield Library Services E-Repository 